\title{
Light Pulses Change Speed in a Plasma
}

\author{
Interactions between two laser beams in a plasma allow for precise \\ control over the light's velocity.
}

\section{By Rachel Berkowitz}

ight has a strict speed limit, but under certain conditions, this boundary can be broken for individual pulses of light. So far, light has been made to travel above its speed limit in media including atomic gases and optical fibers. Clément Goyon from Lawrence Livermore National Laboratory, California, and colleagues now show how to adjust the speed of light in a plasma, creating an order-of-magnitude speed change of the light. The demonstration could help improve control over inertial-confinement-fusion experiments and could lead to plasma-based optics for high-power lasers.

The velocity at which pulses of light pass through a material can strongly differ from the speed $c$ that light travels in a vacuum. This velocity, called the group velocity, can be both higher or lower $\operatorname{than} c$, and it factors in how the shape of a light pulse spreads and distorts as it moves through a material.

For their demonstration, Goyon and colleagues first created a hydrogen-helium plasma by ionizing a jet of the gas with a

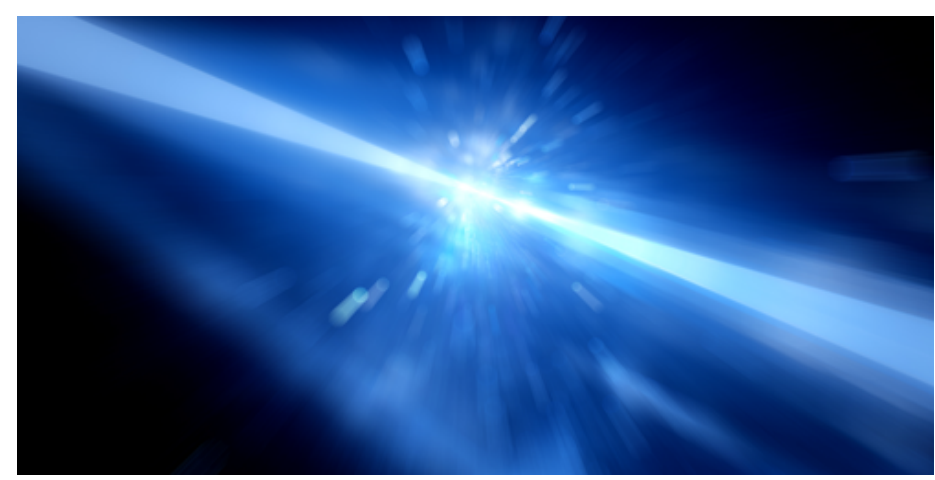

Credit: sakkmesterke/stock.adobe.com polarized laser beam. They then aimed a second laser beam at the plasma. Where the paths of the two beams crossed, the horizontal component of the second laser pulse slowed in response to a change in the plasma's refractive index. This slowing came from interactions between the two lasers and the plasma.

Measuring the time delay between the horizontal and vertical components of the second laser pulse, the team observed that they had different speeds. By tuning the frequency difference between the two beams, they found that they could tune that speed from $0.995 c$ to between $0.12 c$ and $-0.34 c$, indicating that the peak of the pulse traveled faster than $c$.

Rachel Berkowitz is a Corresponding Editor for Physics based in Vancouver, Canada.

\section{REFERENCES}

1. C. Goyon et al., "Slow and fast light in plasma using optical wave mixing," Phys. Rev. Lett. 126, 205001 (2021). 